\title{
Talento humano manufactura de frutas tropicales y la cadena de valor de la industria alimentaria de bebidas no alcohólicas Manabí-Ecuador
}

Fecha de recepción : 15 de noviembre de 2019 • Fecha de aceptación: 18 de marzo de 2020 • Fecha de publicación: 10 de mayo de 2020

Carlos Arturo Monar Merchán'1 Universidad Nacional Mayor de San Marcos - Perú carlos.monar@gmail.com

https://orcid.org/0000-0002-8639-0964

Edgar Vicente Armas ${ }^{2}$ Universidad Nacional Mayor de San Marcos - Perú edgarvicente02@gmail.com https://orcid.org/0000-0003-3112-5247

Pedro Leonardo Tito-Huamaní ${ }^{3}$ Universidad Nacional Mayor de San Marcos - Perú ptitoh@unmsm.edu.pe https://orcid.org/0000-0002-2989-9203

\section{Resumen}

La alimentación es parte del buen vivir del ser humano, luego de la convergencia del Estado, Academia y Empresa. La teoría de la cadena de valor permitió detectar la existencia de una brecha en la formación del talento humano dentro de la manufactura de frutas tropicales. El objetivo de este artículo fue determinar si el talento humano de la manufactura de frutas tropicales tiene relación e incide en la industria alimentaria de bebidas no alcohólicas en la provincia de Manabí-Ecuador. La metodología empleada se alinea a una investigación de tipo cuantitativa analítica, el diseño correlacional ha medido el grado de incidencia; los instrumentos para el levantamiento de datos fue un cuestionario, aplicado mediante una encuesta y la opinión de expertos actores de la Cadena Productiva y registra- 
dos en la base de datos del Servicio de Rentas Internas del Ecuador (SRI), codificados en la Clasificación Industrial Internacional Unificada (CIIU), productores, fabricantes y comercializadores de las bebidas no alcohólicas. Los resultados reflejan que la formación del talento humano si influye significativamente en la Cadena de Valor de la Industria Alimentaria de Bebidas no Alcohólicas, del análisis realizado, la significación 0,00 es menor a 0,05, e incluso menor a 0,01, lo que demuestra que la correlación (grado de incidencia) que se ha establecido, es cierta.

Palabras clave: manufactura, frutas tropicales, cadena de valor, industria alimentaria, talento humano.

\begin{abstract}
Food is part of the good life of the human being, after the convergence of the State, Academy and Company. The value chain theory, its analysis allowed to detect the existence of a gap in the training of human talent within the manufacture of tropical fruits. The objective of this study is to determine if the human talent of the manufacture of tropical fruits is related and influences the food industry of non-alcoholic beverages in the province of Manabí-Ecuador. The methodology used is aligned to an analytical quantitative research, the correlation design has measured the degree of incidence; the instruments for data collection was a questionnaire, applied through a survey and the opinion of expert actors in the Productive Chain and registered in the database of the Internal Revenue Service of Ecuador (SRI), encoded in the International Unified Industrial Classification (CIIU), producers, manufacturers and marketers of non-alcoholic beverages. The results say that the training of human talent does have a significant influence on the Value Chain of the Food Industry of Non-Alcoholic Beverages in Manabí-Ecuador. From the analysis carried out, the significance of 0.00 is less than 0.05 , and even less than 0.01 , which shows that the correlation (degree of incidence) that has been established is true.
\end{abstract}

Keywords: manufacture, tropical fruits, value chain, food industry, human talent. 


\section{Introducción}

En la provincia de Manabí-Ecuador, el talento humano que se encuentra inmerso en la actividad de la industria manufacturera, según la Secretaría Nacional de Planificación y Desarrollo del Ecuador (SENPLADES), en la Agenda Zona 4 del Pacífico (2017), significa un 11\% de la población, ya sea en el campo de las ingenierías e industrias, en donde el tercer nivel alcanza el 3.844 graduados (Manabí 3.838 y Santo Domingo 6), el cuarto nivel alcanzan el 164 graduados (Manabí 158 Santo Domingo 6), entre las carreras, del Sistema Nacional de Educación Superior del Ecuador (SNIESE-2015); es decir, la Zona 4 representa el 9,75\% y 7,66\% respectivamente del total de la población formada en educación superior en Ingenierías. Si consideramos que la Industria Manufacturera de Alimentos y Bebidas, según la revista especializada en industrias Ekos $\mathrm{N}^{\circ} 286$ (2018), representa el $38 \%$ de las Industrias, quiere decir, que este talento humano en la actividad manufacturera y la industria alimentaria sea bajo, apenas el 6,6\%, por lo que no ha incidido en la productividad y desarrollo de esta.

En ese contexto, en las intervenciones de los gobiernos del Ecuador se han identificado entre las causas principales del subdesarrollo la histórica polarización del sector manufacturero concentrándose en 3 ciudades: Quito, Guayaquil y Cuenca. En el 2008, Guayas, Pichincha y Azuay tenían el $86 \%$ de las empresas activas del país, así lo expresó en su debido momento el Ministerio Coordinador de la Política Económica y Comercial del Ecuador; por lo que obviamente la provincia de Manabí no aparece ni en las estadísticas regionales. Según la Senescyt 2018, el 70\% de oferta de cuarto nivel se concentra en la Región Sierra (Quito y Cuenca) y apenas el $30 \%$ en la Región Costa (Guayaquil).

No obstante, en el contexto mundial y regional externo se habla que el desarrollo de los países se lo construye desde los territorios o regiones internas, explica José Antonio Ocampo (2011), quien además termina señalando que, el desarrollo es la capacidad de una economía para generar innovaciones y emprendimientos sectoriales dinámicas. Es decir, que resulta imperativo el cambio de política pública respecto a la formación del talento humano, hacia dar facilidades a las provincias.

La Organización de las Naciones Unidas (ONU) señala que "las interconexiones entre demanda, inversión, comercio y productividad se pueden continuar aún con la ausencia de esfuerzos de políticas públicas y privadas; sin embargo, esto impediría el progreso de los Objetivos de Desarrollo Sostenibles" (ONU, 2017, pág. 1).

Basado en lo anterior se podría profundizar, por ejemplo, en la desnutrición crónica de los niños, debido a los pobres niveles de educación y por ende desarrollo económico. Ana Afonso (2016) sostiene que "en Manabí existe una falta de recursos familiares como indicador renta que incide como factor para la desnutrición crónica de niños"; niños que serán el futuro "factor clave para el desarrollo regional y la competitividad empresarial, como sistema social" (Osorio, 2008, p.1). Por lo que, si sigue igual, Manabí estará lejos del nuevo paradigma, donde su talento humano poco incidirá en el desarrollo económico, pues la desnutrición infantil es la "causa de la reducción irreversible en su rendimiento escolar y luego en el rendimiento laboral" (Afonso et al, 2016, p.261). 
En las economías regionales, aún con potencialidades económicas, sin talento humano competente, su desarrollo será desigual, tanto en las cadenas productivas y sectores industriales, como en nuestro país, la manufactura representan el 17.3\% del PIB según el Banco Central del Ecuador; dentro de la cual, la industria de alimentos y bebidas tiene el $38 \%$ de participación en el sector manufacturero (...) no ha podido internacionalizarse" (EKOS-286, 2018, p.58).

Las cifras de la provincia de Manabí para el análisis territorial resultan interesantes, por ejemplo, "el 15,58\% de la población tiene instrucción superior. La población dedicada a la actividad manufacturera es baja, apenas llega al 8,2\%, de la PEA" (Senplades, 2013-2017, p. 47). Según el Plan de Desarrollo y Ordenamiento Territorial de la Provincia de Manabí (2017), "la mayoría de empresas están en actividades primarias y emplean al $46,8 \%$ de hombres y $24,2 \%$ de mujeres; las actividades secundarias apenas emplean al $18,5 \%$ de hombres y el $31.7 \%$ de mujeres" (GPM, 2017, p. 139).

Consecutivamente se puede señalar que el talento humano actual de la Provincia de Manabí no responde a las necesidades territoriales, y peor a los problemas y sus causas de las empresas, pues "los empleados requieren una serie de aptitudes y talentos extras para ser productivos, que los vuelvan más competitivos, ya que, con un mayor nivel y calidad de servicio, existirá un mayor nivel de competencia de los empleados" (Murillo, 2014, p.10).

El desarrollo del talento humano en la historia del hombre no es un misterio, pues en la práctica social se miden los avances de las sociedades de los territorios, por eso al tratarse de la provincia de Manabí, en el contexto ecuatoriano, debería ser el interés del Estado a través de sus Gobiernos Subnacionales, y sean estos quienes aborden este tema mediante la apertura de formación a través de políticas públicas.

En esta línea, existen factores alimentarios en la formación del talento humano de un territorio y comienza con la adecuada nutrición en la niñez, ya desde el año 2002 miembros de la Universidad Autónoma de México señalaron que "los progresos en la educación y el mejoramiento de la salud física y mental están directamente relacionados con el grado de nutrición; la orientación alimentaria tiende a cambiar la conducta del humano" sostienen Navarro \& Navarro, y se confirma la regla con la "relación entre el estado nutricional y la categoría de coeficiente intelectual, la misma que es directamente proporcional y estadísticamente significativa” (Mamani et al, 2014, p.9).

A pesar de lo antes señalado, en nuestra provincia y en otros territorios pequeños se presentan situaciones similares y no tienen respuesta alguna ante la presencia de evidencias de "desnutrición crónica, siendo este un problema de fondo y que está afectando a uno de cada cuatro niños menores de cinco años, causando una reducción irreversible en su rendimiento escolar y en su futuro rendimiento laboral que merece primordial atención" (Afonso et al, 2016, p. 161).

En segundo lugar, desde lo práctico, existen necesidades de emprendimientos en la industria alimentaria de bebidas no alcohólicas y su cadena de valor, que deben generar una visión a largo plazo, con una estrategia de diferenciación, costos o focalizándose siempre las potencialidades territoriales, como es el caso de las frutas tropicales, cuya principal función es convertirse en antioxidantes beneficiosos para el ser humano, ya sea en salud o nutrición. 
Las frutas tropicales son reconocidas mundialmente, no solo por su crecimiento de la producción y consumo según la FAO (2017), sino por ser ricas en compuestos bioactivos principalmente carotenoides, flavonoides, ácidos fenólicos, estilbenos y taninos con gran potencial antioxidante" (Cárdenas et al, 2015, p.38); siendo una de las cadenas productivas más representativas de la provincia del mercado local, nacional e internacional.

Y en tercer lugar, existe la incidencia del talento humano en la manufactura de frutas tropicales y por ende en la Cadena del Valor de la Industria Alimentaria de Bebidas no Alcohólicas de Manabí, cuya actividad económica analizada es a la transformación de frutas como la naranja, mango, aguacate, papaya, zapote, maracuyá, guanábana, guayaba, limón, entre otras. Por lo que, el objetivo de esta investigación fue determinar la incidencia de la formación del talento humano de la manufactura de frutas tropicales en la cadena de valor.

\section{El talento humano}

El talento humano es el verdadero capital que tienen las organizaciones para generar una gestión del conocimiento, son las personas con gran "actividad, información, experiencias, motivación, intereses, aptitudes, habilidades, potencialidades" (Vallejo, 2015, p.19), las que logran una comprensión organizacional, es decir, poseen "algún diferencial competitivo que representan cuatro aspectos esenciales, conocimiento, habilidad, juicio y actitud, que debe integrarse al activo de las organizaciones" (Chiavenato, 2009, pp. 49-50).

Por lo que, "existe una correlación entre los resultados económicos con las capacidades de formación profesional y el conocimiento global disponible a nivel local, de igual manera, se afirma que los países centrados en la competitividad del talento humano tienden a obtener mejores resultados" (Chávez y Vizcaíno, 2017, p.16).

\subsection{Manufactura de Frutas Tropicales}

Las frutas tropicales son aquellos que nacen, desarrollan y se forman en las zonas tropicales y con clima cálido, están cercanas a la zona ecuatorial con temperaturas por encima de los $18^{\circ} \mathrm{C} 1$; estas frutas desarrollan características nutricionales y gustativas, por ejemplo, en el mango, guayaba, guanábana, papaya y aguacate son parte importante de la oferta exportable de nuestros países ubicados en la zona tórrida del planeta.

Ya casi al finalizar el siglo pasado se creía que la manufactura era exclusivamente aquella actividad de transformación de bienes, capital o productivos, pese a esto, la manufactura hoy también se la relaciona con la transformación de alimentos.

"Es un mecanismo para el cambio de estado y forma de materiales en artículos útiles para la sociedad" (Erazo, 2008, p.3). También se considera una "fuente de ventaja competitiva, es la transformadora de materia prima rápida, fácil y económica; con niveles de servicio al cliente" (Mital, et al, 2017, p.8). Se puede decir entonces que estudia a los procesos o etapas de transformación

Fuente: https://www.caracteristicas.co/clima-tropical/\#ixzz5XDnjkQFr 
en las empresas y que disponen de materias primas, insumos y tecnología, para convertirlo en un producto deseado por una demanda.

Tenemos entonces, una industria manufacturera y un mercado de alimentos y bebidas no alcohólicas que en los primeros años del milenio, se ha posicionado en todos los países del mundo:

Ahora, "es altamente dinámico, y obedece a tendencias globales de consumo. Uno de los motivadores ha sido el generar relacionamiento con la salud. El mercado de las bebidas, las orientadas a la salud y el bienestar; según Euromonitor, agrega las categorías de bebidas, energizantes y deportivas, lácteas (yogur y leches saborizadas), jugos y néctares, té y café y aguas embotelladas. Se incluyen todas las bebidas que expresan proclamas a favor de la salud y el bienestar" (Castrillón, 2018, pp. 5-6). En este contexto esta industria de bebidas no alcohólicas, por ejemplo, "en el mercado mundial en 2015 se registró ventas por un valor de USD 615,0 mil millones y un crecimiento promedio anual de 3,3\% entre 2011 y 2015" (CCC², 2016, p.2).

\subsection{Cadenas de valor (CDV)}

Los aportes de algunos autores, mismos que hacen mención relevante sobre la cadena de valor en la industria de alimentos y bebidas no alcohólicas, que relacionando con la aportación de Michael Porter (2008), se considera un factor de competitividad, él señala que, "es el análisis del costo estratégico que implica la comparación de los costos unitarios entre compañías competidoras claves y de cada actividad (...)" (Quintero \& Sánchez, 2006, p. 380).

En esta misma línea, Marysela Morillo y Albert Márquez señalan la existencia de la cadena de valor agregado como "el conjunto de actividades, desde la entrada de la materia prima hasta la llegada al cliente final" y la cadena de valor industrial como el "conjunto de actividades interconectadas, creadoras de valor, que van desde la obtención de materias primas, hasta que el producto terminado llegue al consumidor final” (Morillo \& Márquez, 2014, p.56).

Sobre esta base se ha ilustrado a la cadena de valor de la industria de alimentos y bebidas no alcohólicas en Manabí como una nobel industria en desarrollo (ver Figura 1).

\section{Metodología}

El estudio es de tipo "cuantitativa analítico, desde el punto de vista del diseño de investigación", ya que se analiza el efecto o influencia de la variable independiente sobre la variable dependiente (Hernández Sampieri, Fernández Collado, \& Baptista Lucio, 2014). 


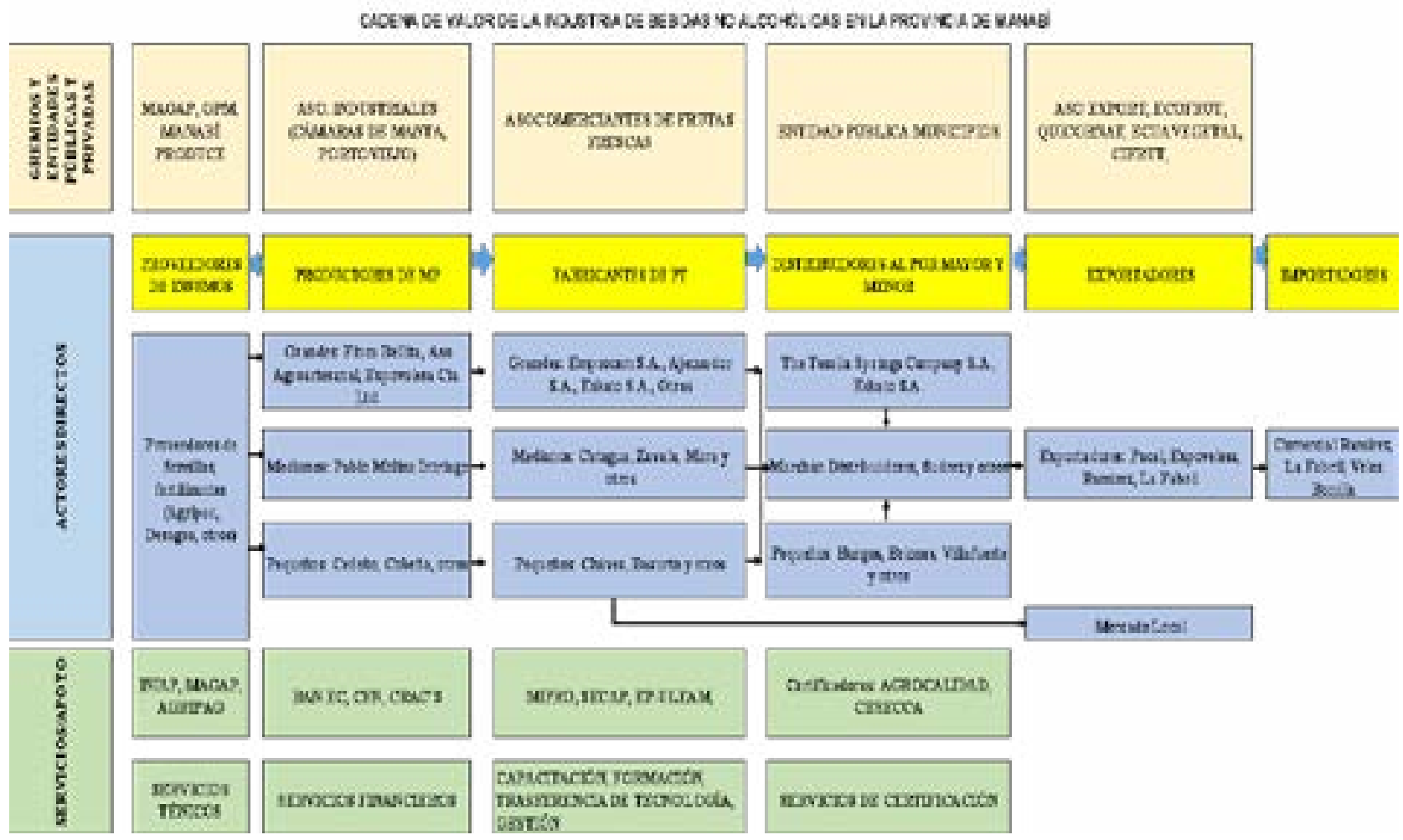

Figura 1. Cadena de valor de bebidas no alcohólicas en Manabí. Fuente: Diagnóstico del Plan de Ordenamiento Territorial de la Provincia de Manabí. GPM. 2017.

Fuente: elaboración propia

La población es la cantidad de actores directos que intervienen en la manufactura de frutas tropicales y la cadena de valor de la industria alimentaria de bebidas no alcohólicas en la provincia de Manabí-Ecuador, cuyo alcance no solo estuvo dentro del territorio manabita, se tomó la base de datos de los contribuyentes al Servicio de Rentas Internas (SRI) del Ecuador con la correspondencia sujeta a la Clasificación Industrial Internacional Unificada (CIIU), misma que al año 2016 ascendía a 25.015 actores relacionados con esta actividad. Los actores directos son los productores, fabricantes y canales de comercialización de frutas tropicales y bebidas no alcohólicas. Los actores indirectos son quienes prestan servicios de apoyo en la cadena de valor.

Se aplicó el cálculo de una muestra probabilística estratificada (Tabla 1), que "consideran segmentos, grupos o estratos" (Hernández et al, 2014). El cálculo se realizó mediante la fórmula para poblaciones finitas: $n=\left(Z^{\wedge} 2 P Q N\right) /\left(Z^{\wedge} 2 P Q \neg+N e^{\wedge} 2\right)$

En donde: $\mathrm{n}=$ tamaño de la muestra; $\mathrm{Z}=$ Nivel de confianza (1.96); $\mathrm{P}=$ Probabilidad de ocurrencia (0.5); $\mathrm{Q}=$ Probabilidad de no ocurrencia (0.5); N= Población = 25.015; Ee= Error de Muestreo (5\%). Para calcular el tamaño de la muestra se consideraron los siguientes datos: Tamaño del universo: 25.015 contribuyentes dedicados a la industria; error máximo aceptable: $5 \%$; porcentaje estimado de la muestra: 50\%; nivel deseado de confianza: 95\%; se estratificó la población para aumentar la precisión de la muestra, se utiliza la siguiente fórmula (Hernández et al, 2014): Ksh =nh/Nh; nh = tamaño de la muestra; Nh = población total. Muestra 379/25.015 =KornShell (ksh) 0,0151509095 
Tabla 1.

Muestra probabilística estratificada

\begin{tabular}{llll}
\hline No. & ACTORES & POBLACIÓN & MUESTRA \\
\hline 1 & $\begin{array}{l}\text { Productores de frutas e insumos en general para la industria de } \\
\text { bebidas no alcohólicas }\end{array}$ & 87 & 1 \\
\hline 2 & $\begin{array}{l}\text { Productores de Frutas tropicales (Maracuyá, Mango, Plátano, } \\
\text { Guanábana, Guayaba) }\end{array}$ & 7.240 & 110 \\
& Empresas Industriales de Productos Terminados & 87 & 3 \\
\hline 3 & $\begin{array}{l}\text { Distribuidores al por mayor de Productos Terminados (Bebidas no } \\
\text { alcohólicas) }\end{array}$ & 226 & 263,5 \\
\hline 5 & $\begin{array}{l}\text { Distribuidores al por menor de Productos Terminados (Bebidas no } \\
\text { alcohólicas. Mipymes al detalle) }\end{array}$ & 17.375 & 379 \\
\hline
\end{tabular}

Fuente: elaboración propia / Base de datos del SRI

Tabla 2.

Muestra probabilística estratificada según el tipo de empresa y según el porcentaje sobre el total de la población.

\begin{tabular}{lll}
\hline \multicolumn{1}{c}{ Tipo de empresa } & Frecuencia & Porcentaje \\
\hline Producción Insumos & 1 & 0,3 \\
\hline Producción Materias Primas & 110 & 29 \\
\hline Transformación de Bebidas no Alcohólicas (Productos Terminados) & 1 & 0,3 \\
\hline Comercialización al por Mayor & 3 & 0,8 \\
\hline Comercialización al por Menor & 264 & 69,6 \\
\hline Total & 379 & 100 \\
\hline
\end{tabular}

Fuente: elaboración propia

Con la recopilación bibliográfica, de textos, artículos científicos, bases de datos, tesis de maestrías y doctorales, como lo sostiene Ander-Egg (1995), en su debido momento, la finalidad es obtener datos, información y conocimiento, según la necesidad o problema que se estudie. El cuestionario es "un conjunto de preguntas para generar los datos e información sobre el objeto de estudio y el problema principal; considerando las variables a medirse" (Bernal, 2016, p. 250). También, se aplicó la entrevista cualitativa estructurada (Hernández, pág. 199, 2014).

Finalmente, la encuesta utilizó un cuestionario preestablecido en base a la dimensión e indicadores de las variables de la investigación, Héctor Martínez y Lourdes Benítez (2015), le consideran "un instrumento para obtener una muestra representativa de la información del objeto de la investigación, con preguntas destinadas a ser contestadas por un gran número de individuos". Los encuestadores, fueron extensionistas del Gobierno Provincial de Manabí, 2017.

La dimensión fue la formación del talento humano de la manufactura de frutas tropicales (variable independiente $\mathrm{X}$ ); y la cadena de valor de la industria alimentaria de bebidas no alcohólicas de 
Manabí, como variable dependiente (Y).

Se aplicó el coeficiente de Cronbach para validar la confiabilidad del cuestionario que utilizó una escala de tipo Likert. Entonces, la Tabla 3 muestra la fiabilidad del Alfa de Cronbach de 0,967 para los ítems del cuestionario, el resultado del coeficiente indicó una alta fiabilidad.

Tabla 3.

Fiabilidad Alfa de Cronbach

\begin{tabular}{ll}
\hline Estadísticos de fiabilidad & \\
\hline Alfa de Cronbach & No. de elementos \\
\hline 0,967 & 50 \\
\hline
\end{tabular}

Fuente: elaboración propia

\section{Resultados}

En la Tabla 4 se aprecia que, del total de encuestados $n=379$ (100\%), el 0,8\% está completamente en desacuerdo acerca la formación de los recursos humanos en la Manufactura frutas tropicales, el $3,4 \%$ está en desacuerdo, asimismo se aprecia que el 8,2\% está en parte de desacuerdo, mientras que el $46,7 \%$ está parte de acuerdo sobre la formación de los recursos humanos en la Manufactura frutas tropicales. Del mismo modo el 30,9\% está de acuerdo acerca la formación de los recursos humanos en la Manufactura frutas tropicales, y el 10\% está completamente de acuerdo sobre la formación de los recursos humanos en la Manufactura frutas tropicales.

Tabla 4

La formación de los recursos humanos en la Manufactura frutas tropicales

\begin{tabular}{lll}
\hline Categoría & $\mathrm{n}$ & $\%$ \\
\hline Completamente en desacuerdo & 3 & 0,8 \\
\hline En desacuerdo & 13 & 3,4 \\
\hline Parte en desacuerdo & 31 & 8,2 \\
\hline Parte de acuerdo & 177 & 46,7 \\
\hline De acuerdo & 117 & 30,9 \\
\hline Completamente de acuerdo & 38 & 10,0 \\
\hline Total & 379 & 100,0 \\
\hline
\end{tabular}

Fuente: elaboración propia 


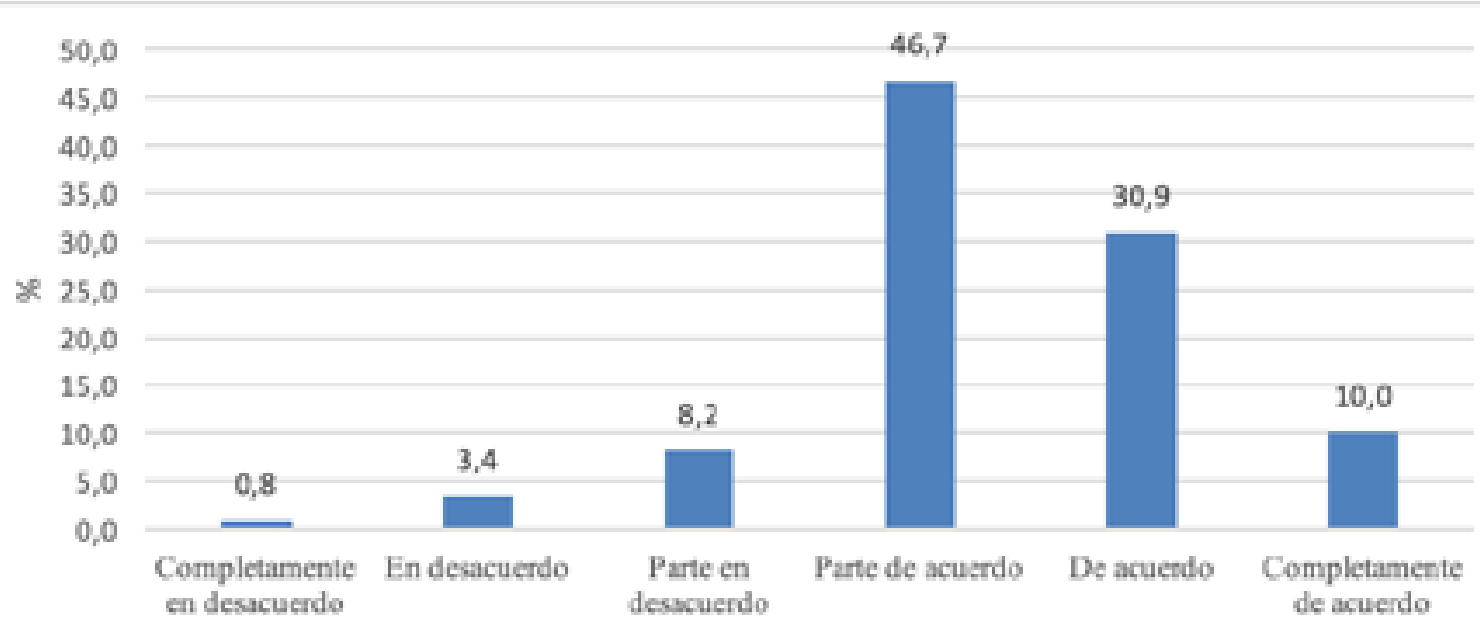

Figura 2. La formación de los recursos humanos en la Manufactura frutas tropicales

Fuente: elaboración propia

De la Tabla 4 y Figura 2 se aprecia que, del total de encuestados $n=379(100 \%)$, el 0,8\% está completamente en desacuerdo acerca la formación de los recursos humanos en la Manufactura frutas tropicales, el 3,4\% está en desacuerdo, asimismo se aprecia que el 8,2\% está en parte de desacuerdo, mientras que el $46,7 \%$ está parte de acuerdo sobre la formación de los recursos humanos en la Manufactura frutas tropicales. Del mismo modo el 30,9\% está de acuerdo acerca la formación de los recursos humanos en la manufactura frutas tropicales, y el 10\% están completamente de acuerdo sobre la formación de los recursos humanos en la Manufactura frutas tropicales. Lo que establece que existe consistencia en la investigación porque ilustra la c normal.

\section{Hipótesis de investigación}

HO: No Existe una relación de la formación del talento humano de la manufactura de frutas tropicales y la cadena de valor de la industria alimentaria de bebidas no alcohólicas en ManabíEcuador.

H1: Existe una relación de la formación del talento humano de la manufactura de frutas tropicales y la cadena de valor de la industria alimentaria de bebidas no alcohólicas en Manabí-Ecuador. Nivel de significación 0,05 
Tabla 5.

Coeficiente de correlación de Spearman entre La formación de los recursos humanos en la Manufactura frutas tropicales. y la cadena de valor

\begin{tabular}{|c|c|c|c|c|}
\hline & & & $\begin{array}{l}\text { La formación de los recursos } \\
\text { humanos en la Manufactura frutas } \\
\text { tropicales. }\end{array}$ & $\begin{array}{l}\text { Cadena } \\
\text { de valor }\end{array}$ \\
\hline \multirow{6}{*}{ 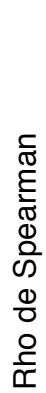 } & \multirow{3}{*}{$\begin{array}{l}\text { La formación de los } \\
\text { recursos humanos } \\
\text { en la Manufactura } \\
\text { frutas tropicales }\end{array}$} & $\begin{array}{l}\text { Coeficiente de } \\
\text { correlación }\end{array}$ & 1,000 &, $597^{\star \star}$ \\
\hline & & $\mathrm{P}$ & & 0,000 \\
\hline & & $\mathrm{N}$ & 379 & 379 \\
\hline & \multirow[t]{3}{*}{ Cadena de valor } & $\begin{array}{l}\text { Coeficiente de } \\
\text { correlación }\end{array}$ &, $597^{\star \star}$ & 1,000 \\
\hline & & $\mathrm{P}$ & 0,000 & \\
\hline & & $\mathrm{N}$ & 379 & 379 \\
\hline
\end{tabular}

Fuente: elaboración propia

Regla de decisión Rechazar Ho si p<0.05 entonces rechazar Ho.

Dado que $\mathrm{p}=0.000<0.05$ entonces rechazar Ho, por lo tanto, aceptar H1, es decir Existe una relación de la formación del talento humano de la manufactura de frutas tropicales y la cadena de valor de la industria alimentaria de bebidas no alcohólicas en Manabí-Ecuador. Asimismo, se encontró moderada correlación positiva y significativa (0.597) entre La formación de los recursos humanos en la Manufactura frutas tropicales y la cadena de valor de la industria alimentaria de bebidas no alcohólicas en Manabí-Ecuador.

La importancia del consumo de frutas es vital debido a sus propiedades nutritivas, ya sea por su función en la prevención de enfermedades crónicas o como por nutrición, su historial de consumo así lo evidencia. Nuestros resultados reportan que existe una relación entre la manufactura de frutas tropicales y la cadena de valor de la industria alimentaria de bebidas no alcohólicas en Manabí-Ecuador.

De esta manera, el $41,2 \%$ está en parte de acuerdo sobre la manufactura de frutas tropicales. EI $43,5 \%$ está de acuerdo acerca de la actividad manufactura de frutas tropicales, el 47,5\% está parte de acuerdo sobre la manufactura de frutas tropicales; $y$, de ese modo el $45,6 \%$ está de acuerdo acerca la cadena de valor.

La cadena de valor hoy se ha tornado una categoría científica de importancia en el mundo del conocimiento, ya que se la nombra no solamente desde la ciencia administrativa, por tal razón, es necesario centrar el enfoque teórico con la debida correspondencia a la temática de este trabajo.

Según la CEPAL (2014) y el proyecto Inserción de Pymes Agroindustriales en las Cadenas Globales de Valor en Centroamérica, esta "comprende la variedad de actividades que se requieren para que un producto o servicio transite a través de las diferentes etapas de producción, desde su concepción hasta su entrega a los consumidores y la disposición final después de su uso". 
De esta manera, los encuestados consideran importante la manufactura de frutas tropicales y su impacto en la cadena de valor, estos resultados coinciden con la investigación Cedeño y Cerón, (2018) quienes establecen el impacto real de la industrialización de frutas tropicales en el desarrollo socioeconómico de los productores del cantón Flavio Alfaro, con la finalidad de determinar la situación de la industria dedicada a la producción de frutas tropicales, realizando un diagnóstico socioeconómico de los productores de ese cantón y analizando el impacto que pueda generar su industrialización.

Los resultados de Cedeño y Cerón (2018) arrojaron que el cultivo de frutas tropicales predominante en la región son la naranja, la mandarina y el mango, motivado fundamentalmente por la demanda que tienen, así como la facilidad que ofrece la zona para su producción. Otra conclusión relevante es la posibilidad de que los agricultores puedan industrializar su producto de forma gradual atendiendo a los requerimientos económicos que pesan sobre este proceso de industrialización.

A su vez, lo obtenido se sustenta por Mahattanatawee (2006), ya que el autor sostiene que estas frutas son beneficiosas para la salud por poseer gran cantidad de antioxidantes naturales y fibra dietética, por lo que protegen el daño celular, mejora la digestión y mantiene los niveles de azúcar en la sangre, además de tener un alto nivel de antioxidantes por los compuestos fenólicos que son sustancias biológicamente activas, y que contribuyen como potencial quimiopreventivo.

Asimismo, se encontró moderada correlación positiva y significativa (0.597) entre la formación de los recursos humanos en la Manufactura frutas tropicales y la cadena de valor de la industria alimentaria de bebidas no alcohólicas en Manabí-Ecuador. Del mismo modo se aprecia que el $46,7 \%$ está parte de acuerdo sobre la formación de los recursos humanos en la Manufactura frutas tropicales; el 30,9\% están de acuerdo acerca de la formación de los recursos humanos en la Manufactura frutas tropicales.

De esta manera, la dimensión del talento humano, como recurso en la fabricación de productos, es uno de los puntos críticos desde el enfoque administrativo, porque sus indicadores se ven afectados cuando el nivel es bajo. Se ha considerado como ejemplo un análisis del sector alimentos y bebidas del estudio de bioeconomía, realizado por Mary Luz Castrillón de la Corporación Biointropic de Medellín Colombia.

\section{Conclusiones}

Cuando hablamos de frutas tropicales se puede afirmar que "ante el rápido crecimiento de la demanda mundial, los países productores han seguido aumentando la superficie dedicada a la producción de frutas tropicales, en especial en los países desarrollados", se debe al incremento experimentado por nuevos mercados y al progreso de la innovación tecnológica de la industria (Altendorf, 2017, p. 3).

La producción de alimentos está regulada por normas y leyes que el estado propone para garantizar la inocuidad de los alimentos que abarca la infraestructura, el procesamiento, personal, proveedores, almacenamiento y reparto del producto terminado, adicionalmente, cada institución 
puede crear parámetros o reglamentos para sus productos o procesos dirigidos a satisfacer a sus clientes.

Por tanto, es importante tener un sistema que garantice que todo lo descrito se cumpla; en este sentido, las buenas prácticas de manufactura deben aplicarse en la elaboración del alimento, dado a que incluye los parámetros a seguir para asegurar que la inocuidad y la calidad del producto elaborado sea óptimo. Por otro lado, el Control Estadístico de Procesos constituye una metodología para asegurar que cierto proceso esté siendo ejecutado apropiadamente y si existe una variabilidad en las especificaciones de control, se puedan realizar las acciones correctivas oportunamente.

Los recursos empleados en la formación del talento humano, siempre son parte del registro de gasto, principalmente en los indicadores de capacitación e investigación, que de paso en nuestras empresas son los menos puntuados; como hemos visto, al tener una relación directa en la CdV de la IAdBnA, al gestarse nuevas cadenas de valor en la industria alimentaria de bebidas no alcohólicas, se podría cambiar ese paradigma y considerar al talento humano como el capital humano o intelectual de las organizaciones, así lo dice Idalberto Chiavenato (2009) y todo capital genera renta y el humano genera desarrollo, más aún si está dentro de una industria en crecimiento y que genera la prevención de "enfermedades catastróficas" (Tobar et al, 2014, p.16). 


\section{Referencias bibliográficas}

Afonso, A (2016), Monar, C., Montalvo, V. Gestión y prioridades geográficas por sectores en Manabí, para mejorar la seguridad alimentaria y desarrollo. Revista ECA Sinergia. UTM. Portoviejo Ecuador.

Bernal, C. (2016). Metodología de la Investigación. Administración, economía, humanidades y ciencias sociales. Tercera Edición. Bogotá D.C. Colombia. Editorial Pearsons.

Cámara de Comercio de Cali, (2016). Informes Económico \#73. Dinámica mundial de la Industria de bebidas no alcohólicas. Cali-Colombia. Recuperado de: www.ccc.org.co/file/2016/04/Informe-N73-EC-Que-hay-parala-sed.pdf

Cárdenas, G. (2015), Arrazola, G. Villalba, M. Frutas tropicales: fuente de compuestos bioactivos naturales en la industria de alimentos, Revista Ingenium de la Universidad de Córdova Argentina, Facultad de ingeniería de Alimentos. Indexada en Dialnet, vol. 17, №33, pp. 29-40, julio de 2015. Recuperado de: https://dialnet. unirioja.es/descarga/articulo/5327083.pdf

Castrillón, M. (2018). Estudio sobre la Bioeconomía como fuente de nuevas industrias basadas en el capital natural de Colombia. Corporación Biointropic. Medellín Colombia. Recuperado de: https://www.dnp.gov. co/Crecimiento-Verde/Documents/ejes-tematicos/Bioeconomia/Informe\%202/ANEXO\%202 An\%C3\%A1lisis\%20sector\%20alimentos\%20y\%20bebidas.pdf

Cedeño, J. Cerón, O. (2018). Industrialización de frutas tropicales: impacto y desarrollo socioeconómico de los productores del Cantón Flavio Alfaro-Ecuador, Revista Observatorio de la

Economía Latinoamericana, Ecuador, (enero 2018). Recuperado de: http://www.eumed.net/cursecon/ecolat/ ec/2018/frutas-tropicales-ecuador.html

CEPAL. (2014). Fortalecimiento de las cadenas de valor como instrumento de la política industrial Metodología y experiencia de la CEPAL en Centroamérica. Editor Ramón Padilla Pérez. Cooperación Alemana. Comisión Económica para América Latina y el Caribe. Santiago de Chile.

Chávez, E. (2017), Vizcaíno, A., Talento humano: una contribución a la competitividad organizacional. Recuperado de: https://dialnet.unirioja.es/descarga/articulo/6067382.pdf

Chiavenato, I. 2009. Gestión del Talento Humano. Tercera edición. Editorial McGraw-Hill/Interamericana Editores, S.A. México, D. F.

EKOS (2018), Industria alimentos y bebidas. Revista especializada. N²86. Recuperado de: https://www.ekosnegocios.com/articulo/industria-de-alimentos-y-bebidas-la-mayor-industria-del-pais

FAO. (2017). Principales frutas tropicales. Recuperado de: http://www.fao.org/3/ca7566en/ca7566en.pdf 
GPM. (2017). Plan de Desarrollo Ordenamiento Territorial. Gobierno Provincial de Manabí (GPM). Portoviejo Ecuador. Recuperado de: https://issuu.com/gadmanabi/docs/pdyot 20manabi 20actualizado

Hernández Sampieri, R. (2014). Metodología de la Investigación. Sexta Edición. Editorial McGraw-Hill / Interamericana Editores S.A. de C.V. Ciudad de México-México. Koontz, H. 2004, Weirich, Heinz, Administración. Una perspectiva global, México. 12ª . Edición. Mc Graw Hill.

Mamani, Y. (2014), Choque M., Rojas E. Estado nutricional y su relación con el coeficiente intelectual de niños en edad escolar. Universidad Mayor de San Simón; Cochabamba - Bolivia. Recuperado en: https://www. researchgate.net/publication/317529136

Martínez, H. (2016), Benítez, L. Metodología de la investigación social I, Cengage Learning Editores, S.A. de C.V., una Compañía de Cengage Learning, Inc. Cruz Manca, Santa Fe C.P. México, D.F

Mital, A. (2017), Noriega, S., López, F., Castaño, V. Ingeniería de Manufactura en el siglo XXI. Un enfoque Estructural para Desarrollo, Diseño y Manufactura de Productos de Consumo. University of Cincinnati, Universidad Autónoma de Ciudad Juárez, Universidad Nacional Autónoma de México. Ed. Academia de Ingeniería, Ciudad de México.

Morillo, M., Márquez, A. (2014). Análisis de la cadena de valor en el sector alimentos y bebidas de los municipios Libertador y Campo Mérida, Venezuela. Agroalimentaria, vol. 20, núm. 38. Redalyc.

Murillo, M. (2014). El talento humano en beneficio de los usuarios del Centro de Salud Andrés de Vera del Cantón Portoviejo. Tesis de Maestría. Universidad Católica de Santiago de Guayaquil Neven, D., 2015, Desarrollo de Cadenas de Valor Alimentarias Sostenibles. Principios Rectores, Organización de las Naciones Unidas para la Alimentación y la Agricultura, Roma.

Porter, M. (2008). Estrategia Competitiva. Técnicas para el análisis de los sectores industriales y de la competencia. 38ava reimpresión, Grupo Editorial Patria. México.

Senplades (2013-2017). Agenda Zonal Zona 4-Pacífico. Provincias de: Manabí y Santo Domingo de los Tsáchilas. Recuperado de: https://www.planificacion.gob.ec/wp-content/uploads/downloads/2015/11/Agenda-zona-4.pdf

SNIESE. (2015). Número de titulados, Clasificados según los campos del conocimiento 2010-2015. Sistema Nacional de la Educación Superior del Ecuador. Recuperado de: http://www.senescyt.gob.ec/visorgeografico/

Tobar, F. (2014), Bürgin, M., Drago, G., Lifschitz, E., Yjilioff, A. Respuestas a las enfermedades catastróficas, 1ra edición. Buenos Aires: Fundación CIPPEC. Recuperado de: https://www.cippec.org/wp-content/ uploads/2017/03/1283.pdf

Vallejo, L. 2015. Gestión del Talento Humano. Instituto de Investigaciones. Escuela Superior Politécnica de Chimborazo. Riobamba, Ecuador. 
Copyright (c) 2020 Carlos Arturo Monar Merchán, Edgar Vicente Armas y Pedro Leonardo Tito-Huamaní

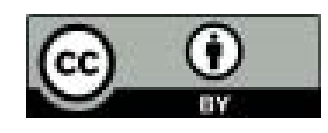

Este texto está protegido bajo una licencia internacional Creative Commons 4.0.

Usted es libre para Compartir-copiar y redistribuir el material en cualquier medio o formato - y Adaptar el documento - remezclar, transformar y crear a partir del material-para cualquier propósito, incluso para fines comerciales, siempre que cumpla las condiciones de Atribución. Usted debe dar crédito a la obra original de manera adecuada, proporcionar un enlace a la licencia, e indicar si se han realizado cambios. Puede hacerlo en cualquier forma razonable, pero no de forma tal que sugiera que tiene el apoyo del licenciante o lo recibe por el uso que hace de la obra.

Resumen de licencia - Texto completo de la licencia 